\title{
Gemcitabine inhibits the micrometastasis of non-small cell lung cancer by targeting the EpCAM-positive circulating tumor cells via the HGF/cMET pathway
}

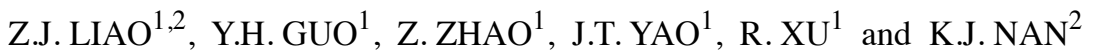 \\ ${ }^{1}$ Department of Medical Oncology, Shaanxi Province Tumor Hospital; ${ }^{2}$ Department of Medical Oncology, \\ The First Affiliated Hospital of The School of Medicine of Xi'an Jiaotong University, \\ Xi'an, Shaanxi 710061, P.R. China
}

Received February 28, 2014; Accepted May 2, 2014

DOI: 10.3892/ijo.2014.2464

\begin{abstract}
Recurrence and metastasis are responsible for the death of non-small cell lung cancer (NSCLC) patients. Circulating tumor cells (CTCs) in the metastatic pathway have proven to be essential. This pilot study evaluated the sensitivity of gemcitabine in micrometastasis and CTCs from NSCLC patients. EpCAM-positive CTCs were detected in forty patients with NSCLC at treatment initiation and disease evaluation time-points. EpCAM-positive CTCs were defined as EpCAM-positive and CD45-negative. Total RNA was isolated from EpCAM-enriched CTCs and cytokeratin levels were detected by PCR. The HGF/cMET pathway was evaluated in CTCs from patients with different treatments and in A549 cells. The EMT-related markers were analyzed by IHC. We further explored the predictive value of baseline CTCs in patients that were receiving different treatments. The median number of CTCs in NSCLC patients was $65 \mathrm{CTCs} / \mathrm{ml}$ more than in the healthy 23 -fold (median, $5.2 \mathrm{CTCs} / \mathrm{ml}$ ). The mean change in cell count was significantly different for patients with gemcitabine compared to patients with non-gemcitabine treatments $(-86.28$ vs. $-15.23 / \mathrm{ml} ; \mathrm{P}<0.05)$. A significant decrease was noted in the expression of cytokeratin in the CTCs of the two groups $(\mathrm{P}<0.05)$. The HGF/cMET pathway was inactivated in CTCs and A549 cells treated with gemcitabine, and the cell migration and invasion abilities were inhibited by gemcitabine via the HGF/cMET pathway. Furthermore, the decreased cell migration and invasion abilities may also be involved in the inhibition of the epithelial-mesenchymal transition (EMT) by gemcitabine. At a median follow-up of 36 months, the CTC count was confirmed to be a robust prognostic marker in the
\end{abstract}

Correspondence to: Professor K.J. Nan, Department of Medical Oncology, The First Affiliated Hospital of The School of Medicine of Xi'an Jiaotong University, Xi'an, Shaanxi 710061, P.R. China E-mail: nkjxjtu@163.com

Key words: gemcitabine, non-small cell lung cancer, circulating tumor cell, EpCAM, HGF/cMET pathway
NSCLC population (CTCs $>151$, median: 15.0 months and CTCs $<151$, median: 32.0 months). Additionally, the survival rate in the gemcitabine group ( 24 months) was better than in non-gemcitabine group (21 months), suggesting a therapeutic benefit for NSCLC patient survival with the common therapy plus gemcitabine. Gemcitabine treatment decreased EpCAMpositive CTCs in NSCLC patients and inhibited EMT by the HGF/cMET pathway.

\section{Introduction}

Lung cancer is the leading cause of cancer-related death worldwide. Approximately, $85 \%$ of lung cancer cases are nonsmall cell lung cancer (NSCLC), which has a more variable behavior and depends on histological type (1). Additionally, more than $40 \%$ of these patients will have distant metastasis outside of the chest at the time of diagnosis. Circulating tumor cells (CTCs) are cells that have shed into the vasculature from a primary tumor, circulate in the bloodstream and are seeds for the subsequent growth of additional tumors, also called metastasis to vital distant organs. CTCs trigger a mechanism that is responsible for the vast majority of cancer-related deaths (2-5). Their role in the metastatic pathway has proven to be essential.

However, CTCs are rare, representing as few as one cell per $10^{9}$ hematologic cells in the blood of patients with metastatic cancer; hence, their isolation presents a tremendous technical challenge (6,7). Microfluidic-based devices (called the CTC-chips) provide unique opportunities to isolate, quantify and analyze circulating tumor cells from a blood sample (8-10). An average of 132 EpCAM-positive circulating tumor cells per milliliter (median, 67 cells $/ \mathrm{ml}$ ) are isolated at high purity from virtually all tested patients with metastatic cancers, but not from healthy controls $(10,11)$. The FDA-approved CellSearch system has set the standard for the use of EpCAM in the enrichment of CTCs using a magnetic ferrofluid approach (12-15). EpCAM is also used as a main capture component in other immunomagnetic bead-based systems as well as microfluidic systems $(16,17)$. A number of studies using the CellSearch system have shown a good correlation between the numbers of these circulating EpCAM-positive cells and the prognosis for cancer survival $(18,19)$. 
However, the development of metastasis depends on multiple factors that determine overall tumor cell growth, survival, angiogenesis and invasion (20). For epithelial malignancies, the epithelial-mesenchymal transition (EMT) is considered the crucial event in the metastatic process, which involves the disruption of epithelial cell homeostasis and the acquisition of a migratory mesenchymal phenotype allowing these cells to travel to the site of metastasis formation without being affected by conventional treatment (21). Accordingly, through mesenchymal-to-epithelial transition (MET), the opposite of the EMT, a metastasis occurs followed by a micrometastasis $(22,23)$. The EMT appears to be controlled by signal-transduction pathways such as the Wnt (24), transforming growth factor $\beta$ (TGF- $\beta$ ) (25) and HGF/cMET (26) pathways, all of which can be aberrantly activated during neoplasia (27-29).

Gemcitabine is a nucleoside analog (30). As a chemical drug, gemcitabine replaces one of the nucleic acids during DNA replication to arrest the tumor growth as only one additional nucleoside can be attached to the 'faulty' nucleoside, resulting in apoptosis (31). Gemcitabine has been used in various carcinomas: non-small cell lung cancer, pancreatic cancer, bladder cancer, breast cancer and other tumor types (32-35). GemCarbo chemotherapy, consisting of a combination of gemcitabine and carboplatin, is used to treat several different types of cancer, but is most commonly used to treat later period non-small cell lung cancer $(36,37)$. However, the therapy indication of gemcitabine for NSCLC with micrometastasis is still unknown. The mechanism of action of gemcitabine as a chemotherapy drug for NSCLC is also not fully understood.

Here, we detected the EpCAM-positive CTCs of NSCLC patients before and after the gemcitabine treatment. Then, other common clinical parameters and survival rates were followed up. The mechanism study showed that gemcitabine targeted the EpCAM-positive CTCs, inhibiting metastasis and invasion by inverting the EMT features induced by the HGF/cMET pathway in NSCLC. These results suggested that gemcitabine chemotherapy can effectively inhibit metastasis and circulating tumor cells in non-small cell lung cancer.

\section{Materials and methods}

Patient eligibility and study design. Forty patients aged 45-75 years, pathologically diagnosed, without obvious distant metastases and in clinical stages II and III of NSCLC regardless of surgical treatment were enrolled in this study. Patients were randomly divided into two groups of experimental and control. Eligibility criteria included hemoglobin level $\geq 8 \mathrm{~g} / \mathrm{dl}$. Patients were ineligible if enrolled in a concurrent treatment protocol in which the total weekly blood draw would exceed $150 \mathrm{ml}$. The negative control samples were obtained from healthy adults. Patients provided written informed consent and the protocol was approved by the Cancer Research Center of Shaanxi Province.

All patients were required to have disease evaluations at 3-week intervals. The experimental group patients underwent systemic gemcitabine therapy for 63 days in 3 periods. The control group was treated with a palliative or curative resection. Twenty milliliters of peripheral blood were obtained from patients at study entry and at 3-week intervals to correspond with points of disease evaluation by computed tomography (CT) scan. After chemotherapy, all patients were rechecked and followed for 3 years.

During the period of recruitment, each subject was scheduled for an interview after written informed consent was given and a structured questionnaire was administered by the interviewer to collect information about demographics and risk factors such as smoking status and alcohol use. The population study was approved by the institutional review board 'Ethics Committee of Shaanxi Province Tumor Hospital' in Shannxi, China. The Ethics Committee of Shaanxi Province Tumor Hospital approved the design of the NSCLC study including samples collection.

Cell culture and drug. The human non-small cell lung cancer cell lines A549, NCI-H460, HCC827 and NCI-H1299 were purchased from ATCC (American Type Culture Collection, Manassas, VA, USA). Cells were cultivated in RPMI-1640 medium (Sigma-Aldrich, St. Louis, MO), with the appropriate amount of heat-inactivated fetal bovine serum (FBS, Invitrogen, Carlsbad, CA) at $37^{\circ} \mathrm{C}$ in an atmosphere of $5 \% \mathrm{CO}_{2}$. All of the experiments were performed during the cell growing to exponential phase and after a culture confluence of $80-90 \%$.

For the first line of chemotherapy, all the patients received platinum-based combination chemotherapy. The chemotherapeutic agent added to the platinum was gemcitabine (Lilly, Suresnes, France) at the recommended dose of $1250 \mathrm{mg} / \mathrm{m}^{2}$ for the 28 experimental group patients. In one treatment period of 21 days, the treatment was administered to patients in the first and eighth day for three periods. For the cell line, gemcitabine was added at a concentration of $50 \mathrm{nmol} / \mathrm{l}$ and the culture media was replaced every day. HGF agent was purchased from Sigma Co. and added to the A549 cells at a concentration of $50 \mu \mathrm{g} / 1$.

Isolation and enrichment of CTC. To isolate CTCs from NSCLC patients, whole peripheral blood cells $(10 \mathrm{ml})$ were centrifuged with Ficoll-Hypaque, a solution with a density of $1.077 \mathrm{~g} / \mathrm{ml}$. Because red blood cells and granulocytes have densities $>1.077 \mathrm{~g} / \mathrm{ml}$ and mononuclear cells have a density $<1.077 \mathrm{~g} / \mathrm{ml}$, centrifugation utilizing Ficoll-Hypaque helps create a layered separation of these cell types. Mononuclear cells, lymphocytes, platelets and granulocytes were collected after centrifugation and washed twice with PBS. Cells were then incubated at $4^{\circ} \mathrm{C}$ for 30 min with EpCAM immunomagnetic fluid. Following incubation, the sample was placed in a magnetic field, selected and washed with PBS. Isolated NSCLC CTCs were then divided and cultured with RPMI-1640 medium.

Flow cytometry. To determine the percentage of CTCs in the peripheral blood of NSCLC patients, flow cytometry was performed. Blood samples $(2-3 \mathrm{ml})$ were drawn into Cell Save tubes, which were maintained at room temperature and processed within $72 \mathrm{~h}$ of collection. CTCs were defined as nucleated EpCAM-positive cells, lacking CD45 (BectonDickinson, Franklin Lakes, NJ). All CTC evaluations were performed by flow cytometry (Becton-Dickinson).

Reverse-transcriptase PCR and real-time PCR. Total RNA from cells was extracted using the TRIzol Reagent (Invitrogen). Total cDNA was used as a template for amplification at $95^{\circ} \mathrm{C}$ 
for 5 min followed by 30 cycles for EpCAM and 25 cycles for $\beta$-actin as a control. Real-time quantitative PCR was performed in triplicate for each primer set and in each cell sample using an iQ5 multicolor real-time PCR Detection System (Bio-Rad, Hercules, CA). The protocol for real-time PCR was 1 cycle of $95^{\circ} \mathrm{C}$ for $30 \mathrm{sec}, 40$ cycles of $95^{\circ} \mathrm{C}$ for $5 \mathrm{sec}, 60^{\circ} \mathrm{C}$ for $30 \mathrm{sec}$ and then a dissociation stage. The cycle threshold (CT) value was determined as the point at which the fluorescence exceeded a limit preset by the instrument's software. PCR primer sequences for EpCAM, CK8, CK18, CK19 and $\beta$-actin are as follows: EpCAM F: TACACTGCCCAGGAGCCAGA, R: TG GCACCAGTGTCCGGATTA; CK8 F: GCTTCTCCGCTC CTTCTAGGATCT, R: GACACCTTGTAGGACTTCTGG GTCA; CK18 F: AAATCTCAGGACCTCGCCAAG, R: GTC TCAGCAGCTCCAACCTCA; CK19 F: CTGAGTGACA TGCGAAGCCAATA, R: CAGTAACCTCGGACCTGCTC ATC; $\beta$-actin F: CTAAGTCATAGTCCGCCTAGAAGCA, R: TGGCACCCAGCACAATGAA.

Western blot analysis. Western blot analyses were performed as previously described with the lysates from cells. Rabbit polyclonal antibodies against human p-cMET and cMET (1:500 dilution; Santa Cruz Biotechnology, Santa Cruz, CA) and mouse monoclonal antibody against human $\beta$-actin (1:500 dilution; Santa Cruz Biotechnology) were incubated with the membranes at $4^{\circ} \mathrm{C}$ overnight, followed by a secondary incubation using horseradish peroxidase (HRP)-conjugated anti-rabbit or anti-mouse IgG (Thermo Fisher Scientific, Inc., New York, NY). Proteins were briefly incubated with an enhanced chemiluminescence reagent (Millipore, Billerica, MA) and then visualized on X-ray film.

Transwell analysis. For the migration assay, $5 \times 10^{5}$ cells were plated onto a 6-well plate (Corning, Lowell, MA, USA) with an $8-\mu \mathrm{m}$ properly carbonate membrane. For the invasion assay, $5 \times 10^{5}$ cells were placed on plates pre-coated with $20 \mu \mathrm{g}$ Matrigel. In both assays, cells were plated in medium without serum, and medium containing $10 \%$ FBS in the lower chamber served as a chemoattractant. After $24 \mathrm{~h}$, cells that did not migrate or invade through the pores were removed by cotton swabs. The inserts were fixed, stained and three random fields for each insert were counted. The results were averaged among three independent experiments.

Statistical analysis. All clinical data were collected independently by two physicians. The survival analysis was calculated using the Kaplan-Meier product limit method. The log-rank test was used to compare survival between groups, according to the CTC count and the type of systemic therapy. The t-test and the Pearson's $\chi^{2}$ test were used to determine significant differences in patient characteristics according to the baseline CTC count. All statistical analyses, performed using the SPSS 16.0 software (SPSS, Inc., Chicago, IL, USA), were twosided, and $\mathrm{P}<0.05$ was considered statistically significant.

\section{Results}

Patient characteristics. Forty patients were enrolled on this study. Eighteen patients underwent surgery One patient was deemed ineligible after enrollment and did not contribute
Table I. Patient characteristics.

\begin{tabular}{lc}
\hline No. of patients & 39 \\
Age (mean \pm SD) (years) & $63.5 \pm 9.4$ \\
Cender & 16 \\
$\quad$ Female & 23 \\
Male & \\
TNM stage & 21 \\
II & 18 \\
III & \\
Grade & 2 \\
I & 23 \\
II & 14 \\
III & \\
Surgical specimen & \\
Surgery & 18 \\
Non-surgery & 21 \\
\hline
\end{tabular}

any blood specimens. Twenty-one patients had previously received chemotherapy, with eighteen having been treated with gemcitabine. Additional patient characteristics are listed in Table I.

Detection of EpCAM-positive CTCs in non-small cell lung cancer patients. To date, antigen-based CTC isolation strategies have made use of the transmembrane protein EpCAM for cell capture, followed by staining for cytoplasmic keratins (CKs) ubiquitously expressed by all epithelial cell types. In our work, the expression of EpCAM and CD45 was first detected in the 4 NSCLC cell lines A549, NCI-H460, HCC827 and NCI-H1299 by flow cytometry. Nearly all cancer cells were EpCAM positive and CD45 negative (Fig. 1A). Furthermore, quantitative analysis of EpCAM staining counts in NSCLCderived CTCs was performed (Fig. 1B). Both male and female healthy controls had insignificant counts (median, $5.2 \mathrm{CTCs} / \mathrm{ml}$; range, 0-16; mean, 6.6 \pm 1.1 ) (Fig. 1B,b2). For consistent analysis of CTC counts in patients with NSCLC, we set a threshold of detection in patients that was higher than any count noted in any healthy donor. Thirty-two of $37(86 \%)$ patients (median,

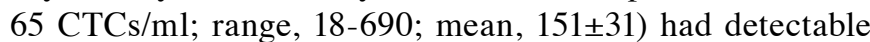
concentrations of CTCs relative to 0 of 16 healthy controls (Fig. 1B,b1). Compared with the healthy controls, the CTCs in the NSCLC patients were almost 23 -fold (Fig. 1B,b3, $\mathrm{P}<0.05$ ).

Next, the EpCAM-expressing CTCs in the NSCLC patients were isolated with EpCAM immunomagnetic fluid. Following isolation, CTCs were divided and cultured with RPMI-1640 medium (Fig. 1C). Then, we detected the expression of EpCAM in isolated CTCs by RT-PCR. The results showed that all of the CTCs isolated by EpCAM beads expressed a considerable amount of EpCAM transcript (Fig. 1D).

Significant decrease of EpCAM-expressing CTCs due to gemcitabine. Gemcitabine has been used in various carcinomas, including non-small cell lung cancer, especially later period 

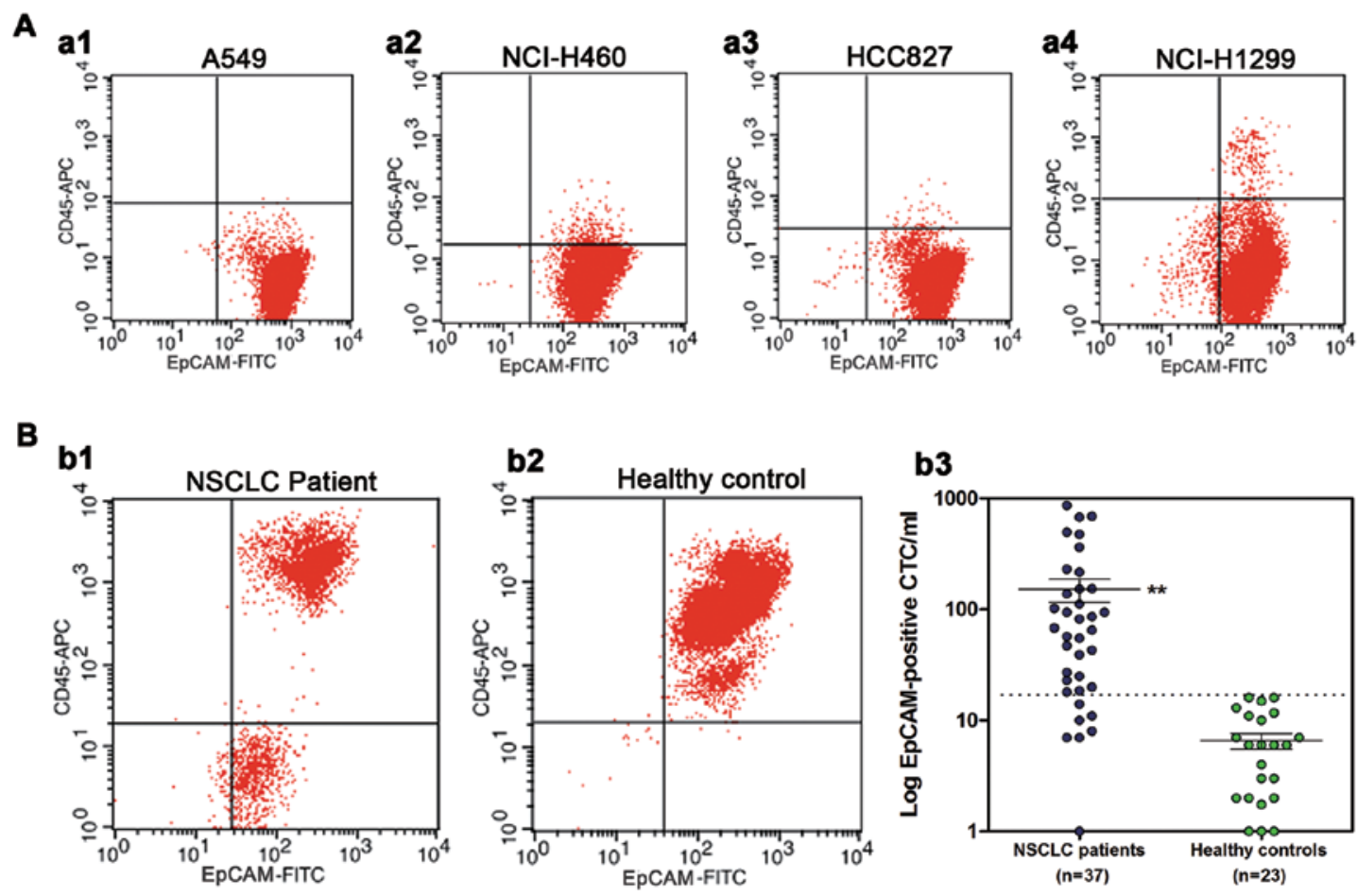

C

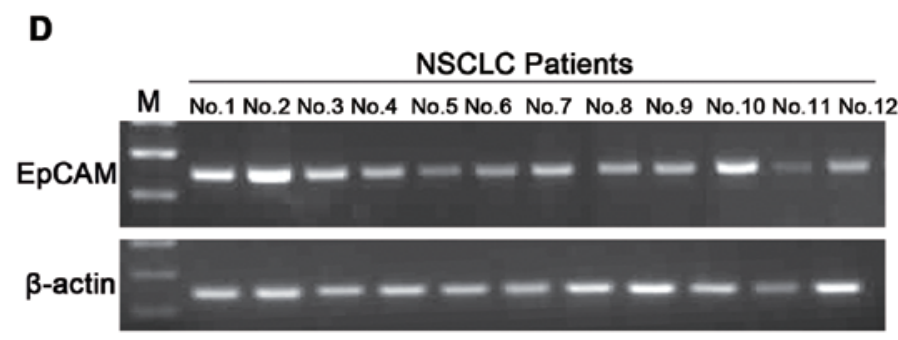

Figure 1. Detection of EpCAM-positive CTCs in NSCLC patients. (A,a1-a4) EpCAM-positive and CD45-negative cells were detected in four NSCLC cell lines A549, NCI-H460, HCC827 and NCI-H1299 by FACS. (B) EpCAM-positive and CD45-negative CTCs were detected in the peripheral blood of 39 NSCLC patients (b1) by FACS; the 24 healthy patients as the control and the statistical results are shown in b2. (C) EpCAM-positive CTCs were isolated and cultured in vitro. (D) The expression of EpCAM mRNA was detected in CTCs derived from NSCLC patient peripheral blood by PCR. Bars, SE. "P $<0.05$.

NSCLC. However, the therapy indication for gemcitabine in NSCLC with micrometastasis is still unknown and the mechanism of action of gemcitabine as a chemotherapy drug in NSCLC is not fully understood. Here, we compared the EpCAM-expressing CTC counts of two groups treated with systemic gemcitabine therapy or a palliative or curative resection. When the EpCAM-expressing CTCs were evaluated at 3 intervals of 63 days, there was a gradual, significant decrease of these cells in the gemcitabine group (mean: from $99.28 \pm 29.00$ to $13.00 \pm 2.84 \mathrm{CTCs} / \mathrm{ml}, \mathrm{P}<0.05$ ); however, in the non-gemcitabine group, the EpCAM-expressing CTCs counts showed no significant change (mean: from $102.23 \pm 19.00$ to $87.00 \pm 2.84$ CTCs $/ \mathrm{ml}, \mathrm{P}>0.05$, Fig. $2 \mathrm{~A}$ ).

Across different patients with different characteristics of the disease (see Table I for patient characteristics), the change in the number of EpCAM-positive CTCs/ml was positively correlated with the TNM stage and differentiation grade, especially in the gemcitabine group. However, it was poorly correlated with surgery (Fig. 2B). Additionally, the expression of cytokeratin, a protein marker for epithelial cells, was used to evaluate the relative CTC yield. Here, the evaluation of the expression levels of selected genes (CK8, CK18 and CK19) based on the RNA extracted from the CTC-enriched baseline blood samples was feasible using quantitative real-time PCR (Fig. 2C). After 3 treatment periods, the gemcitabine group patients with $13.00 \pm 2.84 \mathrm{CTCs} / \mathrm{ml}$ peripheral blood expressed higher CK8, CK18 and CK19 genes compared with the non-gemcitabine group patients with $77.26 \pm 24.51 \mathrm{CTCs} / \mathrm{ml}$ (Fig. $2 \mathrm{C}$ ). These results suggested that treatment with gemcitabine in NSCLC patients significantly decreased the EpCAM-positive CTCs and cytokeratin gene expression levels.

HGF/cMET pathway was significantly inhibited by gemcitabine in NSCLC. To explore the pathway involved in the micrometastasis of tumor cells, the HGF level in the peripheral blood serum was gradually decreased along with the treatment of gemcitabine (Fig. 3A). As the receptor for the HGF factor, the activated p-cMET was inhibited by gemcitabine in the EpCAM-positive CTCs; however, for the non-gemcitabine group, neither HGF nor p-cMET were shown to be inhibited (Fig. 3B). Additionally, the HGF/c-MET pathway was indeed inhibited by gemcitabine in the NSCLC cell line A549. The p-cMET gene was inactivated when the cells were treated with gemcitabine, and gemcitabine also inhibited the increase 
A

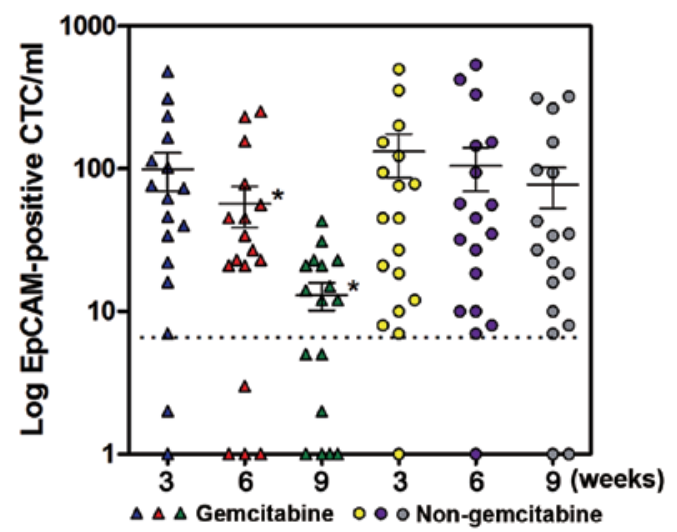

B

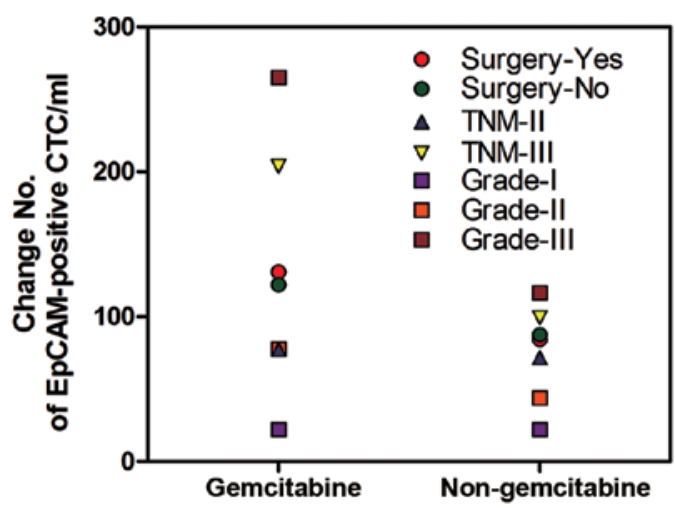

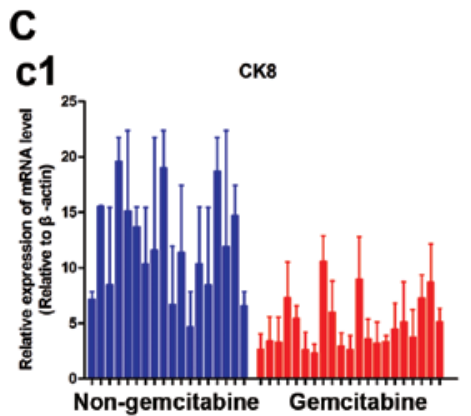
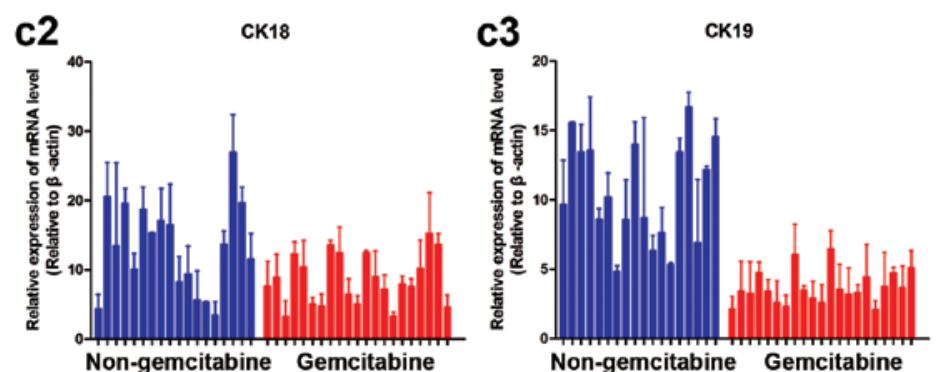

Figure 2. Significant decrease of EpCAM-expressing CTCs due to gemcitabine. (A) The number of EpCAM-expressing CTCs/ml was evaluated at 3 intervals of 63 days treatment in the gemcitabine group and non-gemcitabine group. (B) The number of EpCAM-positive CTCs/ml was valued along with different characteristics of the disease such as TNM stage, differentiation grade and surgery in the gemcitabine group and non-gemcitabine group. (C,c1-c3) The expression of cytokeratin, CK8, CK18 and CK19 was detected in the CTC-enriched baseline blood samples using quantitative real-time PCR.

A

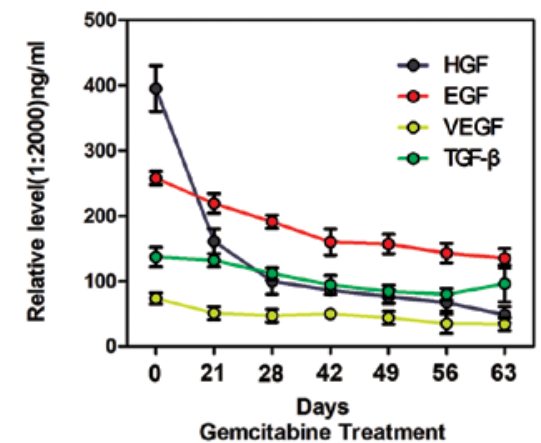

C

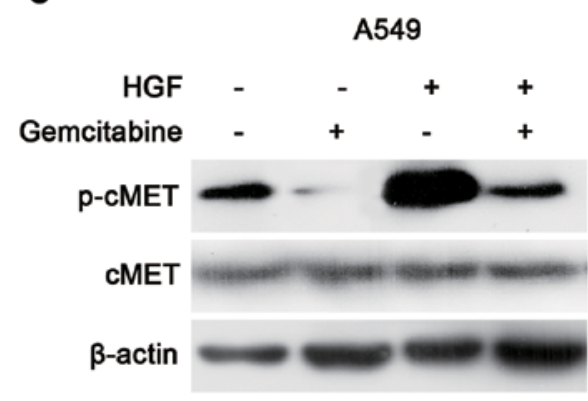

B

EpCAM-positive CTC

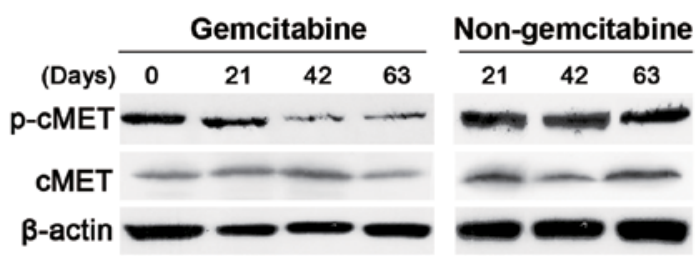

D

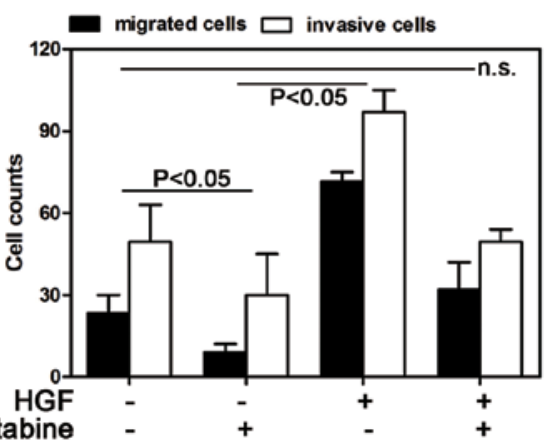

Figure 3. HGF/cMET pathway was significantly inhibited by gemcitabine in NSCLC. (A) The HGF, EGF, VEGF and TGF- $\beta$ relative levels in the peripheral blood serum were detected by EALSA assay at intervals of 63 days of gemcitabine treatment. (B) The activated p-cMET and cMET protein were detected at 3 weeks intervals of 63 days treatment in the gemcitabine group and non-gemcitabine group by western blot analysis. (C) p-cMET and cMET levels were valued in the NSCLC cell line A549 with the conditions of HGF, gemcitabine or both by western blot analysis. (D) The cell migration and invasion abilities were analyzed when A549 cells were treated with HGF, gemcitabine or both by transwell assay. Bars, SE. "P<0.05; ns, P>0.05. 


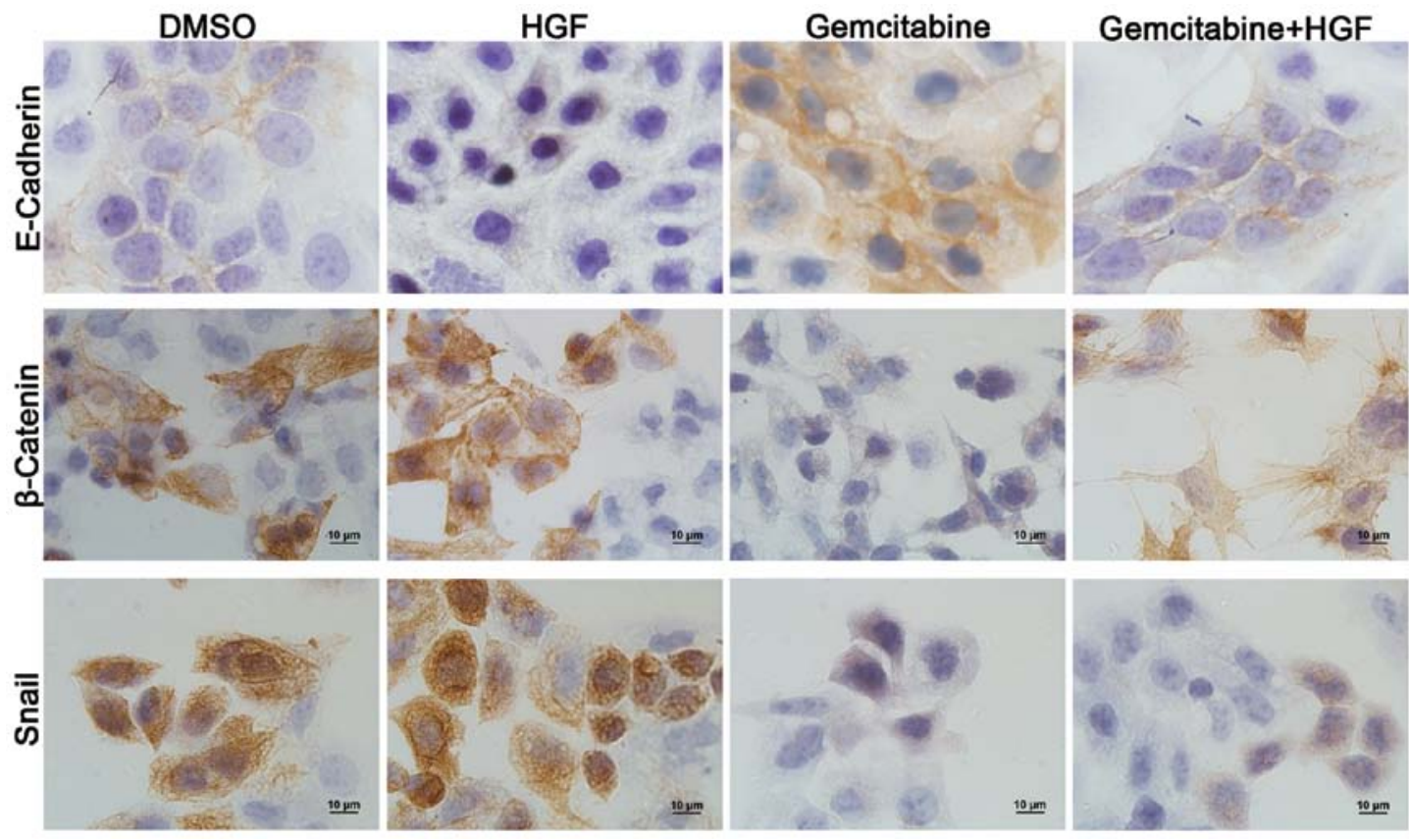

Figure 4. Gemcitabine inhibited cell migration and invasion by EMT. The EMT related markers E-Cadherin, $\beta$-Catenin and Snail were detected in the NSCLC cell line A549 with different treatments of HGF, gemcitabine or both by IHC.
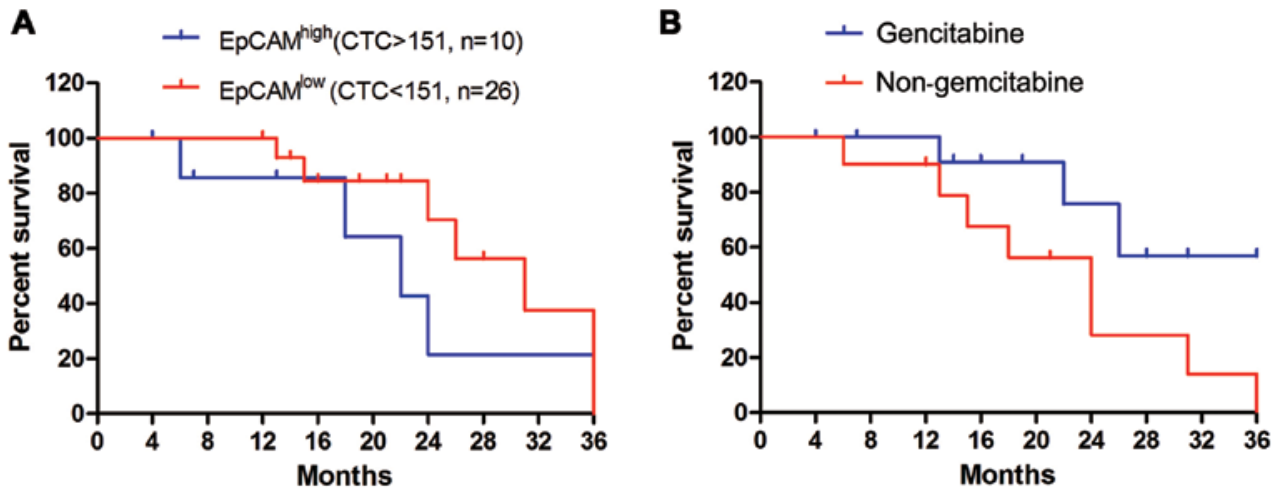

\begin{tabular}{lccl}
\multicolumn{4}{c}{ NSCLC survival (months) } \\
\hline Group & Median & 95\% C.I. & Log-rank P \\
\hline EpCAM $^{\text {high }}$ & 15.0 & $10.19 \sim 26.21$ & $<0.05$ \\
EpCAM $^{\text {low }}$ & 32.0 & $29.43 \sim 34.80$ & \\
\hline
\end{tabular}

\begin{tabular}{|c|c|c|c|}
\hline \multicolumn{4}{|c|}{ NSCLC survival (months) } \\
\hline Group & Median & $95 \%$ C.I. & Log-rank P \\
\hline & 24 & $16.80 \sim 27.09$ & \\
\hline Non-gemcitabine & e 21 & $15.53 \sim 24.59$ & \\
\hline
\end{tabular}

Figure 5. Prognostic value of CTC and gemcitabine treatment in the NSCLC patients. (A) Estimated NSCLC patient survival according to baseline circulating tumor cell (CTC) value ( $<151$ vs. $\geq 151)$ in the overall population. (B) Estimated NSCLC patient survival in the gemcitabine group and non-gemcitabine group. $95 \% \mathrm{CI}, 95 \%$ confidence interval.

of p-cMET binding to HGF (Fig. 3C). Furthermore, the cell migration and invasion abilities were inhibited after treatment with gemcitabine, which was consistent with the decreased activity of the HGF/cMET pathway $(\mathrm{P}<0.05)$. The migrated and invasive cell count was restored when the cells were treated with gemcitabine and HGF, suggesting that gemcitabine effectively inhibited the HGF/c-MET pathway and the cell migration and invasion abilities.

Gemcitabine inhibited cell migration and invasion by epithelialmesenchymal transition. For epithelial malignancies, the EMT is considered to be the crucial event in the metastatic process and appears to be controlled by signal-transduction pathways such as the HGF/cMET pathways. To confirm this, the EMT related markers E-Cadherin, $\beta$-Catenin and Snail were detected in the NSCLC cell line A549 with different treatment by IHC (Fig. 4). The staining results showed that the epithelial marker E-Cadherin was downregulated and mesenchymal markers $\beta$-Catenin and Snail were markedly upregulated at the protein level when cells were cultured in the medium containing HGF. Cells treated with gemcitabine were shown to be undergoing epithelialization with high expression of E-Cadherin and low expression of $\beta$-Catenin and Snail. Gemcitabine could effectively reverse the EMT forced by HGF. This result suggested 
that gemcitabine may inhibit the EMT, resulting in decreased cell migration and invasion abilities.

Prognostic value of circulating tumor cells and gemcitabine treatment in NSCLC patients. The median follow-up time for all patients was three years; 20 patients $(55.56 \%)$ had died at the time of analysis. We found a remarkable correlation between the baseline value of CTCs and the outcome of all patients. The median survival was 15.0 months $(95 \% \mathrm{CI}$ 10.19-26.21) for EpCAM ${ }^{\text {high }}$ patients with CTCs $>151$ and 32.0 months (95\% CI 29.43-34.80) for those EpCAM ${ }^{\text {low }}$ patients with CTCs $<151$ (Fig. 5A, log-rank P<0.05). Additionally, the differential ability of each modality of treatment to reduce the CTC number led us to evaluate whether the gemcitabine therapy could impact the prognostic value associated with a high count of CTCs. We evaluated the survival value in the two treatment groups, including the common chemotherapy (non-gemcitabine group) and common chemotherapy plus gemcitabine (Gemcitabine group). The median survival rate was 21 months (95\% CI 15.53-24.56) for patients treated without gemcitabine and 24 months (95\% CI 16.80-27.09) for those treated with gemcitabine $(\log -$ rank $\mathrm{P}=0.059$, Fig. $5 \mathrm{~B})$. These results suggested a therapeutic benefit for the NSCLC patient survival with the common therapy plus gemcitabine.

\section{Discussion}

The study of CTCs is essential to understanding the vascular spread of cancer to distant sites and for determining the higher risk of cancer progression. Up until now, multistep batch purification strategies such as immunomagnetic bead capture have been used, but microfluidic approaches have the advantage of simpler processing steps and allow the isolation of viable cells at higher sensitivity and purity. Here, we applied the epithelial cell adhesion molecule-associated antigen EpCAM, which is expressed in the vast majority of carcinomas, to isolate the CTCs in NSCLC patient peripheral blood. We detected the EpCAM-positive CTCs and determined the survival rates for the NSCLC patients treated with gemcitabine, exploring the clinical significant and mechanism of action of gemcitabine on micrometastasis in non-small cell lung cancer.

Recurrence and metastasis are the key factors that determine treatment effectiveness and survival time associated with drug resistance or insensitivity of tumor cells, EMT, micrometastasis and so on. In the present study, the rare CTCs in the peripheral blood play an important role in recurrence and metastasis through the formation of distant micrometastases (38). Several studies have confirmed that the number of peripheral blood CTCs was much higher in patients with cancers such as breast, colon and prostate cancer than in healthy patients (3). Furthermore, the CTC level was correlated with the metastasis degree and survival, especially in breast cancer (11). Here, we detected the NSCLC CTCs by staining for EpCAM expression. The threshold CTC number chosen to optimally distinguish between patients with cancer and cancer-free patients $(6.6 \mathrm{CTCs} / \mathrm{ml})$ may underestimate the CTCs in some cancer patients with few true EpCAM-positive cells, but it ensures a low likelihood of false positives. Thirty-two of 37 (86\%) patients (median, $65 \mathrm{CTCs} / \mathrm{ml}$; range, 18-690; mean, $151 \pm 31$ ) had detectable concentrations of CTCs compared to
0 of 16 healthy controls. Compared with the healthy controls, the CTCs in NSCLC patients were almost 23-fold. Although the isolation of CTCs based on EpCAM expression has been established for the detection of breast cancer, colon cancer and NSCLC CTCs, other equally sensitive and specific antibodies could in theory be used for the detection of circulating cells from other cancers, such as PSA staining in prostate cancer (39) and HER2 staining in subsets of breast cancer (40) or neural crest marker staining in melanoma (41).

Gemcitabine is the agent designed with a known molecular target to receive FDA approval for the treatment of lung cancer, yet its activity in micrometastasis is unclear, especially in the subgroup of patients with non-small cell lung cancer. We have identified a significant decrease of the EpCAM-positive CTCs as the molecular correlation to the dramatic responses to gemcitabine by NSCLC patients. The HGF/cMET pathway was shown to be inactivated in the isolated EpCAM-positive CTCs of NSCLC patients and A549 cells that responded to the gemcitabine treatment; the non-gemcitabine CTCs may have had the detected HGF/cMET activity. These results, together with the finding of decreased EpCAM-positive CTCs in the peripheral blood of NSCLC patients who received the gemcitabine treatment, suggest that EpCAM-positive CTCs account for the majority of the responses to gemcitabine reported in clinical studies. Additionally, the mechanism of gemcitabine as an anticancer agent for NSCLC by inhibiting the $\mathrm{HGF} / \mathrm{cMET}$ pathways revealed that the EMT feature was effectively reversed and the cell migration and invasion abilities were decreased in NSCLC cells.

Our data also suggested a potential role for enumeration of CTCs and gemcitabine treatment in predicting disease progression. An increased number of EpCAM-positive CTCs was seen in poor disease progression and a change in the CTC number might predict clinical responses. Since we initiated our pilot study, the results of a multi-center study of CTCs in metastatic breast cancer have been published. Patients with $\geq 151 \mathrm{CTCs} / \mathrm{ml}$ peripheral blood at the baseline and follow-up assessments had shorter overall survival compared with patients with $<151 \mathrm{CTCs} / \mathrm{ml}$ peripheral blood. The patients with gemcitabine treatment had longer survival time compared with the nongemcitabine group. These results led to the USA Food and Drug Administration approval of this technology in metastatic breast cancer, with high reproducibility of the test across multiple laboratories. Thus, proof of CTC enumeration predicting clinical outcome and gemcitabine treatment prolonging the survival time have been demonstrated and supports further clinical evaluation of CTCs and gemcitabine in NSCLC.

This pilot study investigated the EpCAM-positive CTCs in NSCLC patients before and after the treatment with gemcitabine. The mechanism study showed that gemcitabine targeted the EpCAM-positive CTCs, inhibiting metastasis and invasion through reversal of the EMT features induced by the $\mathrm{HGF} / \mathrm{cMET}$ pathway in NSCLC. These results suggested that gemcitabine chemotherapy can effectively inhibit metastasis and circulating tumor cells in non-small cell lung cancer.

\section{Acknowledgements}

This study was supported by a grant to Z.J.L. [2006K09-G4 (4)] from the science and technology project of Shaanxi province: 
the clinical study of gemcitabine on resistant non-small cell lung cancer micrometastasis.

\section{References}

1. Ferlay J, Shin HR, Bray F, Forman D, Mathers C and Parkin DM: Estimates of worldwide burden of cancer in 2008: GLOBOCAN 2008. Int J Cancer 127: 2893-2917, 2010.

2. Ni X, Zhuo M, Su Z, et al: Reproducible copy number variation patterns among single circulating tumor cells of lung cancer patients. Proc Natl Acad Sci USA 110: 21083-21088, 2013.

3. Plaks V, Koopman CD and Werb Z: Cancer. Circulating tumor cells. Science 341: 1186-1188, 2013.

4. Li P, Stratton ZS, Dao M, Ritz J and Huang TJ: Probing circulating tumor cells in microfluidics. Lab Chip 13: 602-609, 2013.

5. Young R, Pailler E, Billiot F, et al: Circulating tumor cells in lung cancer. Acta Cytol 56: 655-660, 2012

6. Liu Y, Qian J, Feng JG, et al: Detection of circulating tumor cells in peripheral blood of colorectal cancer patients without distant organ metastases. Cell Oncol (Dordr) 36: 43-53, 2013.

7. Hou HW, Warkiani ME, Khoo BL, et al: Isolation and retrieval of circulating tumor cells using centrifugal forces. Sci Rep 3 : 1259,2013

8. Huang SB, Wu MH, Lin YH, et al: High-purity and label-free isolation of circulating tumor cells (CTCs) in a microfluidic platform by using optically-induced-dielectrophoretic (ODEP) force. Lab Chip 13: 1371-1383, 2013.

9. Diamond E, Lee GY, Akhtar NH, et al: Isolation and characterization of circulating tumor cells in prostate cancer. Front Oncol 2: 131,2012

10. Dong Y, Skelley AM, Merdek KD, et al: Microfluidics and circulating tumor cells. J Mol Diagn 15: 149-157, 2013.

11. Green TL, Cruse JM and Lewis RE: Circulating tumor cells (CTCs) from metastatic breast cancer patients linked to decreased immune function and response to treatment. Exp Mol Pathol 95: 174-179, 2013.

12. Lin HK, Zheng S, Williams AJ, et al: Portable filter-based microdevice for detection and characterization of circulating tumor cells. Clin Cancer Res 16: 5011-5018, 2010.

13. Alunni-Fabbroni M and Sandri MT: Circulating tumour cells in clinical practice: Methods of detection and possible characterization. Methods 50: 289-297, 2010.

14. Ross JS and Slodkowska EA: Circulating and disseminated tumor cells in the management of breast cancer. Am J Clin Patho 132: 237-245, 2009

15. Amadori A, Rossi E, Zamarchi R, Carli P, Pastorelli D and Jirillo A: Circulating and disseminated tumor cells in the clinical management of breast cancer patients: unanswered questions. Oncology 76: 375-386, 2009.

16. Wen CY, Wu LL, Zhang ZL, et al: Quick-response magnetic nanospheres for rapid, efficient capture and sensitive detection of circulating tumor cells. ACS Nano 8: 941-949, 2014.

17. Chen Q, Ge F, Cui W, et al: Lung cancer circulating tumor cells isolated by the EpCAM-independent enrichment strategy correlate with Cytokeratin 19-derived CYFRA21-1 and pathological staging. Clin Chim Acta 419: 57-61, 2013.

18. Pantel $\mathrm{K}$ and Alix-Panabieres C: Circulating tumour cells in cancer patients: challenges and perspectives. Trends Mol Med 16: 398-406, 2010

19. Sieuwerts AM, Kraan J, Bolt J, et al: Anti-epithelial cell adhesion molecule antibodies and the detection of circulating normal-like breast tumor cells. J Natl Cancer Inst 101: 61-66, 2009.

20. Fidler IJ: The pathogenesis of cancer metastasis: the 'seed and soil' hypothesis revisited. Nat Rev Cancer 3: 453-458, 2003.

21. Thiery JP, Acloque H, Huang RY and Nieto MA: Epithelialmesenchymal transitions in development and disease. Cell 139 871-890, 2009.
22. Hugo H, Ackland ML, Blick T, et al: Epithelial-mesenchymal and mesenchymal-epithelial transitions in carcinoma progression. J Cell Physiol 213: 374-383, 2007.

23. Chaffer CL, Thompson EW and Williams ED: Mesenchymal to epithelial transition in development and disease. Cells Tissues Organs 185: 7-19, 2007.

24. Vincan $\mathrm{E}$ and Barker N: The upstream components of the Wnt signalling pathway in the dynamic EMT and MET associated with colorectal cancer progression. Clin Exp Metastasis 25: 657-663, 2008.

25. Zavadil J: New TGF-beta and Ras crosstalk in EMT. Cell Cycle 8: 184, 2009.

26. Thiery JP: Epithelial-mesenchymal transitions in development and pathologies. Curr Opin Cell Biol 15: 740-746, 2003.

27. Polakis P: The many ways of Wnt in cancer. Curr Opin Genet Dev 17: 45-51, 2007.

28. Akhurst RJ, Fitzpatrick DR, Fowlis DJ, Gatherer D, Millan FA and Slager H: The role of TGF-beta $\mathrm{s}$ in mammalian development and neoplasia. Mol Reprod Dev 32: 127-135, 1992.

29. Zhou HY, Pon YL and Wong AS: HGF/MET signaling in ovarian cancer. Curr Mol Med 8: 469-480, 2008.

30. Gesto DS, Cerqueira NM, Fernandes PA and Ramos MJ: Gemcitabine: a critical nucleoside for cancer therapy. Curr Med Chem 19: 1076-1087, 2012.

31. Muggia F, Diaz I and Peters GJ: Nucleoside and nucleobase analogs in cancer treatment: not only sapacitabine, but also gemcitabine. Expert Opin Investig Drugs 21: 403-408, 2012.

32. Cok G, Goksel T, Soyer S and Aysan T: Effectiveness of gemcitabine as second-line chemotherapy in non-small cell lung cancer. Tuberk Toraks 56: 74-80, 2008.

33. Noble S and Goa KL: Gemcitabine. A review of its pharmacology and clinical potential in non-small cell lung cancer and pancreatic cancer. Drugs 54: 447-472, 1997.

34. Galsky MD, Hahn NM, Powles T, et al: Gemcitabine, Cisplatin, and sunitinib for metastatic urothelial carcinoma and as preoperative therapy for muscle-invasive bladder cancer. Clin Genitourin Cancer 11: 175-181, 2013.

35. Martin M, Ruiz A, Munoz M, et al: Gemcitabine plus vinorelbine versus vinorelbine monotherapy in patients with metastatic breast cancer previously treated with anthracyclines and taxanes: final results of the phase III Spanish Breast Cancer Research Group (GEICAM) trial. Lancet Oncol 8: 219-225, 2007.

36. Reaume MN, Leighl NB, Mittmann N, et al: Economic analysis of a randomized phase III trial of gemcitabine plus vinorelbine compared with cisplatin plus vinorelbine or cisplatin plus gemcitabine for advanced non-small-cell lung cancer (Italian GEMVIN3/NCIC CTG BR14 trial). Lung Cancer 82: 115-120, 2013.

37. Gridelli C, Gallo C, Shepherd FA, et al: Gemcitabine plus vinorelbine compared with cisplatin plus vinorelbine or cisplatin plus gemcitabine for advanced non-small-cell lung cancer: a phase III trial of the Italian GEMVIN Investigators and the National Cancer Institute of Canada Clinical Trials Group. J Clin Oncol 21: 3025-3034, 2003

38. Perlikos F, Harrington KJ and Syrigos KN: Key molecular mechanisms in lung cancer invasion and metastasis: a comprehensive review. Crit Rev Oncol Hematol 87: 1-11, 2013.

39. Stott SL, Lee RJ, Nagrath S, et al: Isolation and characterization of circulating tumor cells from patients with localized and metastatic prostate cancer. Sci Transl Med 2: 25ra23, 2010.

40. Wallwiener M, Hartkopf AD, Baccelli I, et al: The prognostic impact of circulating tumor cells in subtypes of metastatic breast cancer. Breast Cancer Res Treat 137: 503-510, 2013.

41. Ma J, Lin JY, Alloo A, et al: Isolation of tumorigenic circulating melanoma cells. Biochem Biophys Res Commun 402: 711-717, 2010. 